\title{
Severe COVID-19 and coagulopathy: A systematic review and meta-analysis
}

Saikat Mitra, ${ }^{1,2}{ }_{M D}$, Ryan Ruiyang Ling, ${ }^{3}{ }_{M B B S}$, Isabelle Xiaorui Yang, ${ }^{3}{ }_{M B B S}$, Wynne Hsing Poon, ${ }^{3} M B B S$, Chuen Seng Tan, ${ }^{4}{ }_{P h D}$, Paul Monagle, ${ }^{5,6,7}$ MD, Graeme MacLaren, ${ }^{1,3}{ }_{M S c}$, Kollengode Ramanathan, ${ }^{1,3}$ FCICM

\begin{abstract}
Introduction: Coronavirus disease 2019 (COVID-19)-induced coagulopathy (CIC) has been widely reported in the literature. However, the spectrum of abnormalities associated with CIC has been highly variable.

Methods: We conducted a systematic review of the literature (until 1 June 2020) to assess CIC and disease severity during the early COVID-19 pandemic. Primary outcomes were pooled mean differences in platelet count, D-dimer level, prothrombin time, activated partial thromboplastin time (aPTT) and fibrinogen level between non-severe and severe patients, stratified by degree of hypoxaemia or those who died. The risk factors for CIC were analysed. Random-effects meta-analyses and meta-regression were performed using $\mathrm{R}$ version 3.6.1, and certainty of evidence was rated using the Grading of Recommendation, Assessment, Development, and Evaluation approach.

Results: Of the included 5,243 adult COVID-19 patients, patients with severe COVID-19 had a significantly lower platelet count, and higher D-dimer level, prothrombin time and fibrinogen level than non-severe patients. Pooled mean differences in platelet count $\left(-19.7 \times 10^{9} / \mathrm{L}, 95 \%\right.$ confidence interval [CI] -31.7 to -7.6$)$, D-dimer level $(0.8 \mu \mathrm{g} / \mathrm{mL}, 95 \%$ CI $0.5-1.1)$, prothrombin time $(0.4$ second, $95 \%$ CI $0.2-0.6)$ and fibrinogen level $(0.6 \mathrm{~g} / \mathrm{L}, 95 \%$ CI $0.3-0.8)$ were significant between the groups. Platelet count and D-dimer level were significant predictors of disease severity on meta-regression analysis. Older men had higher risks of severe coagulopathic disease.

Conclusion: Significant variability in CIC exists between non-severe and severe patients, with platelet count and D-dimer level correlating with disease severity. Routine monitoring of all coagulation parameters may help to assess CIC and decide on the appropriate management.
\end{abstract}

Ann Acad Med Singap 2021;50:325-35

Keywords: Coagulation parameters, coagulopathy, D-dimer, platelets

\section{INTRODUCTION}

Manifestations of the coronavirus disease 2019 (COVID-19) span a wide clinical spectrum, from asymptomatic carriers to critical illness with a wide range of complications..$^{1,2}$ Our understanding of the pathophysiology of the disease process is still evolving. As part of the host response to viraemia, it has been postulated that coagulopathy may play a pivotal role in the pathogenesis of COVID-19. ${ }^{1}$ Autopsy reports highlighting the presence of pulmonary microemboli, ${ }^{3}$ and clinical manifestations, including massive pulmonary embolism and acute cerebrovascular stroke, have been reported. ${ }^{4}$ Systemic microthrombi formation results from the activation of dysregulated coagulation system triggered by the host response to the virus. ${ }^{5}$ Furthermore, patients with severe forms of the disease may present with consumptive coagulopathy ${ }^{3}$ and bleeding. ${ }^{5}$ This requires exploration of laboratory parameters that could help in prediction of disease progression at an early stage and contribute to improvements in outcome. Markers of the severity of coagulopathy have been investigated in some studies. ${ }^{6,7}$ We hypothesised that

\footnotetext{
${ }^{1}$ Cardiothoracic Intensive Care Unit, National University Heart Centre, National University Hospital, Singapore

${ }^{2}$ Department of Intensive Care, Lyell McEwin Hospital, Adelaide, Australia

${ }^{3}$ Yong Loo Lin School of Medicine, National University of Singapore, Singapore

${ }^{4}$ Saw Swee Hock School of Public Health, National University of Singapore, Singapore

${ }^{5}$ Department of Paediatrics, University of Melbourne, Melbourne, Australia

${ }^{6}$ Department of Clinical Haematology, Royal Children's Hospital, Melbourne, Australia

${ }^{7}$ Haematology Research, Murdoch Children's Research Institute, Melbourne, Australia

Correspondence: Dr Saikat Mitra, Cardiothoracic Intensive Care Unit, National University Heart Centre, Level 9, 1E Kent Ridge Road, Singapore 119228.

Email: Saikat.Mitra@sa.gov.au
} 


\section{CLINICAL IMPACT}

\section{What is New}

- Severe COVID-19 patients had a significantly lower platelet count and a higher D-dimer level, prothrombin time and fibrinogen level than nonsevere patients.

- Decreasing platelet counts and increasing D-dimer levels are associated with disease severity.

\section{Clinical Implications}

- COVID-19-induced coagulopathy is dynamic in nature and serial monitoring of all coagulation parameters may help to assess the disease progression.

severe COVID-19 is associated with coagulopathy and therefore carried out this systematic review and metaanalysis to analyse the coagulation parameters associated with disease severity in COVID-19.

\section{METHODS}

\section{Search strategy and selection criteria}

A systematic search was conducted after registering on PROSPERO register (CRD42020181132). The literature review was conducted according to the Preferred Reporting Items for Systematic Reviews and Meta-Analysis (PRISMA) statement using PubMed, EMBASE, Cochrane and Scopus databases until 1 June 2020. The search strings included the Boolean AND, OR and NOT operators, with the following keywords and their respective variants or derivatives in any relevant combination: COVID-19 OR 2019 novel coronavirus disease OR SARS-CoV-2 OR coronavirus disease-19 AND blood coagulation disorders OR fibrin/fibrinogen degradation products OR platelet OR D-dimer OR fibrinogen OR coagulopathy OR thrombin time OR prothrombin time OR activated partial thromboplastin time OR bleeding time. We included case-control studies, cohort studies, case series (sample size of at least 10 patients) and the studies that mentioned coagulopathy. Studies related to animals, paediatric patients and pregnant patients, letters to the editor, as well as articles published in non-English languages or those published from the same centres and covering the same time period were excluded. A hand search of all relevant studies and their citation lists was performed to identify articles for inclusion. Two reviewers (RRL and IXY) independently screened the articles for eligibility, and any conflicts were resolved by consensus or by a third reviewer (SM).

\section{Data collection}

We extracted details on publication, sample size, study period, geographical region, type of study, demographics, coagulation parameters (platelet count, D-dimer, prothrombin time $[\mathrm{PT}]$, activated partial thromboplastin time [aPTT], fibrinogen) and comorbidities. Patients with severe disease were defined as those who were suffering from hypoxaemia according to the World Health Organization interim guidance, ${ }^{8}$ or Diagnosis and Treatment Protocol for Novel Coronavirus Pneumonia (Trial Version 5, Revised), ${ }^{9}$ or Diagnosis and Treatment Protocol for Novel Coronavirus Pneumonia (Trial Version 7), ${ }^{10}$ or those who died from COVID-19. Hypoxaemia was defined as: (1) respiratory rate of $\geq 30 / \mathrm{min}$; (2) oxygen saturation of $\leq 90 \%{ }^{8}$ or $\leq 93 \% 0^{9,10}$ at rest as measured by pulse oximeter; or (3) ratio of partial pressure of arterial oxygen to fraction of inspired oxygen of $\leq 300 \mathrm{mmHg}$. Coagulopathy for this review was defined as a composite outcome of 1 or more of the following: elevated D-dimer levels, deranged coagulation parameters (PT, aPTT, fibrinogen) and deranged platelet count.

\section{Risk of bias assessment}

Two reviewers (RRL and WHP) independently assessed study eligibility using the Joanna Briggs Institute checklist for prevalence studies, and any conflicts were resolved by discussion or by the third reviewer (SM). Publication bias was assessed using the Egger's regression test.

\section{Statistical analyses}

Statistical analyses were done on $\mathrm{R}$ version 3.6.1 (R Foundation for Statistical Computing, Austria), using the packages meta (version 4.12-0) and dmetar (version 0.0 .9000 ). Our primary outcome was pooled mean differences of coagulation parameters (platelet count, D-dimer, PT, aPTT, fibrinogen) between non-severe and severe cases. Secondary outcomes included possible risk factors correlated with coagulopathy such as patient demographics and disease severity.

We anticipated significant interstudy heterogeneity given the differing standards of care among various hospitals for COVID-19 patients. Therefore, randomeffects meta-analyses (method of DerSimonian and Laird $)^{11}$ were conducted. Dichotomous variables were presented as pooled proportions with their corresponding 95\% confidence intervals (CIs), and pooled odds ratios (ORs) with their corresponding 95\% CIs presented 
whenever applicable. Continuous variables were presented as pooled means or pooled mean differences with their corresponding 95\% CIs, and the means and standard deviations for the continuous variables were pooled from the aggregate data of each study using the methods proposed by Wan et al. ${ }^{12}$

Planned subgroup analyses included the presence of comorbidities (diabetes, hypertension, cardiovascular), and sex (male versus female) in COVID-19 patients reported with coagulopathy. We used the Grading of Recommendation, Assessment, Development, and Evaluation (GRADE) guidance to assess between-study heterogeneity and rated the certainty of evidence using the GRADE approach. ${ }^{13-15}$ We used the GRADEpro Guideline Development Tool (McMaster University and Evidence Prime Inc, Canada) to rate the evidences ${ }^{16}$ and create the GRADE evidence profiles and summary-offindings tables using standardised terms. ${ }^{17,18}$ Leave-oneout sensitivity analysis (LOO) was performed by omitting 1 study at a time to identify outliers or influential studies. For all the outcomes, we presented the post-LOO analysis data whenever applicable. Summary-level meta-regression was conducted when at least 6 studies were available to explore potential sources of heterogeneity or prognostically relevant study-level covariates.

\section{RESULTS}

Our preliminary search identified 1,255 articles. After exclusion of duplicates and conducting initial screening, the full texts of 172 citations were obtained for eligibility (Fig. 1). In total, 26 studies $^{19-44}$ that reported on adult COVID-19 patients with coagulopathy (5,243 patients) were included (Table 1) for systematic review, ${ }^{25,30}$ while 24 studies were included for metaanalysis (5,035 patients). The quality assessment performed using the Joanna Briggs Institute checklists showed that the studies were of the highest quality (score $>6 / 9$ ) despite their observational nature. Twenty studies were from China, while 6 were from the Netherlands, Italy, France, Ireland and the US. 19,21,24,25,30,33

\section{Demographics}

The pooled mean age across the 24 studies was 53.8 years $(95 \%$ CI $51.2-56.4)$. The pooled mean age of non-severe COVID-19 patients (17 studies) was 50.8 years (95\% CI 47.6-53.9), while that of severe patients (21 studies) was 61.1 years (95\% CI 58.4-63.9). The estimated proportion of men across the studies was $54.2 \%$ (95\% CI 51.3-57.2). The pooled proportion of men in the non-severe and severe groups with coagulopathy were $54.1 \%(95 \%$ CI $51.1-57.2)$ and
$62.0 \%$ (95\% CI 56.6-67.2), respectively. Table $2 \mathrm{~A}$ depicts the pooled prevalence of diabetes mellitus (17 studies), hypertension (15 studies) and cardiovascular diseases (12 studies) in the overall population. We found that the pooled prevalence of the different comorbidities was higher in the severe group than in the non-severe group (Table 2A). The pooled prevalence of severe coagulopathic patients from 20 studies was $30.1 \%$ (95\% CI 21.8-39.1).

\section{Primary outcomes}

Table 2A illustrates the pooled estimates of different coagulation parameters between non-severe and severe patients with coagulopathy. There was a significant drop in platelet count (11 studies), while D-dimer levels (15 studies), PT (9 studies) and fibrinogen levels (5 studies) were significantly increased in severe patients (Fig. 2). However, the mean difference in D-dimer levels between severe and non-severe patients showed a significant publication bias (Egger's test, $P<0.001)$. No outliers were detected for D-dimer in the LOO analysis. We found a non-significant increase in aPTT (8 studies) in severe patients with coagulopathy. Our meta-regression analysis demonstrated that a fall in platelet count and a rise in D-dimer levels were significant predictors of disease severity in patients with coagulopathy (Table 2B). PT, aPTT and fibrinogen levels did not show any significant association with disease severity.

\section{Secondary outcomes}

Seventeen studies reported on the significant pooled mean difference in age between the study groups; older age was associated with severe coagulopathic disease (mean difference 10.8 years [95\% CI 8.2-13.4], $P<0.001)$. Men had higher risks of acquiring severe disease with coagulopathy (OR $1.51,95 \%$ CI 1.28 $1.79, P<0.001)$. We also noted that patients with diabetes mellitus (OR 3.09, 95\% CI 1.59-5.99, $P<0.001$ ), hypertension (OR $2.85,95 \%$ CI $1.77-4.58$, $P<0.001)$ and cardiovascular diseases (OR 3.79, 95\% CI 2.07-6.96, $P<0.001$ ) had higher risks of suffering from severe disease with coagulopathy.

\section{Mortality outcome}

The pooled mortality estimated from 15 studies was $14.0 \%(95 \%$ CI $8.4-20.7)$ in the patients with coagulopathy.

\section{Risk of bias}

We assessed the certainty of evidence for all primary outcome measures using the GRADE classification 




Fig. 1. Preferred Reporting Items for Systematic Reviews and Meta-analysis (PRISMA) flowchart for study selection.

(Table 3). The starting certainty for all outcomes was high. We found that the certainty of evidence to be high for mean differences in D-dimer level, platelet count, PT and fibrinogen level. However, the certainty of evidence for mean difference in aPTT was low owing to serious inconsistency and imprecision.

\section{DISCUSSION}

The presence of coagulopathy is one of the leading causes of mortality in patients with COVID-19.,45 In the context of the current COVID-19 pandemic, we conducted this analysis to identify coagulation parameters that could aid in severity stratification and prognostication of the disease progression. Our systematic review and meta-analysis comprehensively examined the differences of coagulation parameters between non-severe and severe COVID-19 patients across 26 studies published from 6 different countries. We found significant pooled mean differences in the blood levels of coagulation parameters (platelet count, D-dimer, PT and fibrinogen) between the 2 groups of COVID-19 patients. Older men were more likely 
Table 1. Summary of studies included in the meta-analysis

\begin{tabular}{|c|c|c|c|c|c|c|c|c|}
\hline \multirow[b]{2}{*}{ Source $^{a}$} & \multirow[b]{2}{*}{ Sample size } & \multirow[b]{2}{*}{ Study type } & \multirow[b]{2}{*}{ Country of study } & \multicolumn{5}{|c|}{ Coagulation parameters $^{\mathrm{b}}$} \\
\hline & & & & Platelet count & D-dimer & PT & aPTT & Fibrinogen \\
\hline Bonetti, ${ }^{19} 2020$ & 144 & Observational & Italy & + & + & + & + & - \\
\hline $\mathrm{Cui}^{20} 2020$ & 81 & Observational & China & + & + & + & + & - \\
\hline Fogarty, ${ }^{21} 2020$ & 83 & Observational & Northern Ireland & + & + & + & + & + \\
\hline $\mathrm{Fu}^{22} 2020$ & 75 & Observational & China & + & + & + & + & + \\
\hline $\mathrm{Gao},{ }^{23} 2020$ & 43 & Observational & China & - & + & + & + & + \\
\hline Helms,${ }^{24} 2020$ & 150 & Observational & France & + & + & + & - & + \\
\hline $\mathrm{Klok}^{25} 2020$ & 184 & Observational & The Netherlands & - & - & + & + & - \\
\hline $\mathrm{Li}^{26}{ }^{2} 2020$ & 74 & Observational & China & - & + & + & + & + \\
\hline Liu J, ${ }^{27} 2020$ & 40 & Observational & China & + & + & + & + & + \\
\hline Liu Y, ${ }^{28} 2020$ & 76 & Observational & China & - & + & + & + & + \\
\hline $\mathrm{Lv},{ }^{29} 2020$ & 354 & Observational & China & - & + & - & - & - \\
\hline Panigada, ${ }^{30} 2020$ & 24 & Observational & Italy & + & + & + & + & + \\
\hline $\mathrm{Qu},{ }^{31} 2020$ & 30 & Observational & China & + & - & - & - & - \\
\hline Sun, ${ }^{32} 2020$ & 116 & Observational & China & + & - & - & - & - \\
\hline Tabatabai, ${ }^{33} 2020$ & 10 & Observational & US & - & + & + & + & + \\
\hline Tang, ${ }^{34} 2020$ & 449 & Observational & China & + & + & + & - & - \\
\hline Wan,,$^{35} 2020$ & 135 & Observational & China & + & + & + & + & - \\
\hline $\mathrm{Wu},{ }^{36} 2020$ & 201 & Observational & China & + & + & + & + & - \\
\hline Yang, ${ }^{37} 2020$ & 1476 & Observational & China & + & - & - & - & - \\
\hline Yao, ${ }^{38} 2020$ & 108 & Observational & China & + & + & - & - & - \\
\hline Zhang G, ${ }^{39} 2020$ & 221 & Observational & China & + & + & + & + & - \\
\hline Zhang $\mathrm{G}^{40} 2020$ & 95 & Observational & China & + & + & - & - & - \\
\hline Zhang J, ${ }^{41} 2020$ & 140 & Observational & China & - & + & - & - & - \\
\hline Zhao, ${ }^{42} 2020$ & 532 & Observational & China & + & - & - & - & - \\
\hline Zheng, ${ }^{43} 2020$ & 99 & Observational & China & - & + & + & - & - \\
\hline $\mathrm{Zou}^{44} 2020$ & 303 & Observational & China & - & + & + & + & + \\
\hline
\end{tabular}

aPTT: activated partial thromboplastin time; PT: prothrombin time

${ }^{a}$ Superscript numbers refer to reference numbers in REFERENCES

b ' +' indicates these data can be extracted from the studies, '-' indicates these data cannot be extracted from the studies

to be severely coagulopathic, and these patients had lower platelet counts but higher PT, D-dimer and fibrinogen levels than non-severe patients. We identified that platelet counts and D-dimer levels correlated well with disease severity. We also noted that patients with comorbidities (diabetes mellitus, hypertension or cardiovascular disease) had a higher likelihood of progression to severe disease with coagulopathy than those without comorbidities.
A recent meta-analysis demonstrated that severe COVID-19 is associated with thrombocytopaenia. ${ }^{6,7}$ While single-centre observational studies have shown no significant differences in platelet count between severe and non-severe patients, ${ }^{46,47}$ our review demonstrated that the cumulative pooled mean difference of platelet count was significant between non-severe and severe patients. Deranged coagulation parameters have been correlated with poor prognosis in COVID-19 patients. ${ }^{48}$ 




Fig. 2. Forest plot showing pooled mean differences of coagulation parameters between non-severe and severe patients. 
Table 2. Pooled estimates and 95\% confidence intervals of (2A) comorbidities and coagulation parameters, and (2B) meta-regression analysis

\begin{tabular}{|c|c|c|c|c|c|}
\hline \multicolumn{6}{|c|}{ 2A. Pooled estimates of comorbidities and coagulation parameters } \\
\hline Diabetes mellitus, $\%$ & $13.3(9.7-17.2)$ & $15.0(11.1-19.4)$ & \multicolumn{2}{|c|}{$6.7(4.3-9.4)$} & \\
\hline Hypertension, \% & $24.6(16.0-34.2)$ & $34.8(28.3-41.5)$ & \multicolumn{2}{|c|}{$12.3(8.2-16.9)$} & \\
\hline Cardiovascular diseases, $\%$ & $12.9(5.9-22.1)$ & $8.5(4.9-12.8)$ & \multicolumn{2}{|c|}{$3.0(1.6-4.7)$} & \\
\hline \multicolumn{6}{|l|}{ Coagulation parameter } \\
\hline Platelets, $\times 10^{9} / \mathrm{L}$ & $191.5(184.0-198.9)$ & $177.1(147.2-207.1)$ & \multicolumn{2}{|c|}{$193.7(183.9-203.4)$} & -19.7 (-31.7 to -7.6$), P=0.001$ \\
\hline $\mathrm{D}$-dimer $(\mu \mathrm{g} / \mathrm{mL})$ & $1.0(0.8-1.1)$ & $1.6(1.3-2.0)$ & \multicolumn{2}{|c|}{$0.6(0.5-0.7)$} & $0.8(0.5-1.1), P<0.001$ \\
\hline PT, seconds & $13.0(12.4-13.7)$ & $13.4(12.5-14.3)$ & \multicolumn{2}{|c|}{$12.5(11.8-13.3)$} & $0.4(0.2-0.6), P<0.001$ \\
\hline aPTT, seconds & $31.3(28.7-34.0)$ & $32.8(30.6-35.0)$ & \multicolumn{2}{|c|}{$31.9(28.4-35.3)$} & 0.5 (-0.6 to 1.7$), P=0.35$ \\
\hline Fibrinogen $(g / L)$ & $4.4(3.3-5.4)$ & $4.3(2.6-6.0)$ & \multicolumn{2}{|c|}{$3.8(1.9-5.6)$} & $0.6(0.3-0.8), P<0.001$ \\
\hline \multicolumn{6}{|l|}{ 2B. Meta-regression analysis } \\
\hline Covariate & Studies & Odds ratio & Lower CI & Upper CI & $P$ value \\
\hline D-dimer & 19 & 1.014 & 1.007 & 1.020 & $<0.001$ \\
\hline Platelet count & 15 & 1.550 & 1.187 & 2.024 & 0.001 \\
\hline PT & 14 & 1.022 & 0.998 & 1.046 & 0.071 \\
\hline aPTT & 13 & 1.001 & 0.912 & 1.098 & 0.991 \\
\hline Fibrinogen & 9 & 0.995 & 0.998 & 1.046 & 0.772 \\
\hline
\end{tabular}

aPTT: activated partial thromboplastin time in seconds; CI: confidence interval; PT: prothrombin time in seconds

Increases in PT and aPTT seen in the severe group are likely multifactorial because of consumption of coagulation factors as the disease worsens, and the presence of lupus-like anticoagulants detected in these patients. ${ }^{49,50}$ Elevated fibrinogen levels seen in COVID-19 patients might be due to an underlying highgrade inflammatory response. ${ }^{45}$ We also postulated that COVID-19 patients may develop hyperfibrinogenaemia as they progress from non-severe to severe disease followed by excessive fibrinolysis, elevated D-dimer and fibrin degradation products. Hyperfibrinogenaemia leads to plasma hyper-viscosity, which in turn potentiates endothelium damage and microvascular thrombosis. ${ }^{45}$

Although the exact mechanism of COVID-19associated coagulopathy is still poorly understood, the intricate interplay between inflammation and thrombosis termed as thrombo-inflammation has been implicated..$^{51}$ It is speculated that the vascular endothelium is damaged by the Severe Acute Respiratory Syndrome Coronavirus 2 (SARS-CoV-2) virus ${ }^{52}$ because of binding of SARS-CoV-2 to angiotensin-converting enzyme 2 receptors, ${ }^{53}$ leading to uninhibited and dysregulated thrombin generation with consumptive coagulopathy. ${ }^{54,55}$
Given the spectrum of coagulation disorders ranging from thrombotic complications to consumptive coagulopathy in patients with severe form of the disease, COVID-19induced coagulopathy (CIC) might be a clinical entity that is distinct from disseminated intravascular coagulation or sepsis-induced coagulopathy. ${ }^{56}$ Our analysis showed that the cut-off limits for platelet count, PT and fibrinogen levels in CIC are quite different from those that define sepsis-induced coagulopathy or disseminated intravascular coagulation. Recently published guidelines endorse early anticoagulation in patients with COVID-19; however, our review demonstrated that CIC could be dynamic as the disease progresses and that anticoagulants in severe disease may be used while monitoring the coagulation profile closely. There is some evidence that full-dose anticoagulation therapy in severe COVID-19 patients may be associated with better survival. ${ }^{57}$ Table 4 summarises plausible theories behind coagulopathy with therapeutic interventions in COVID-19..$^{5,50,58-60}$ However, the true mechanisms of coagulopathy and the pathophysiology of disease progression remain unknown, and urgent mechanistic research to determine these aspects 


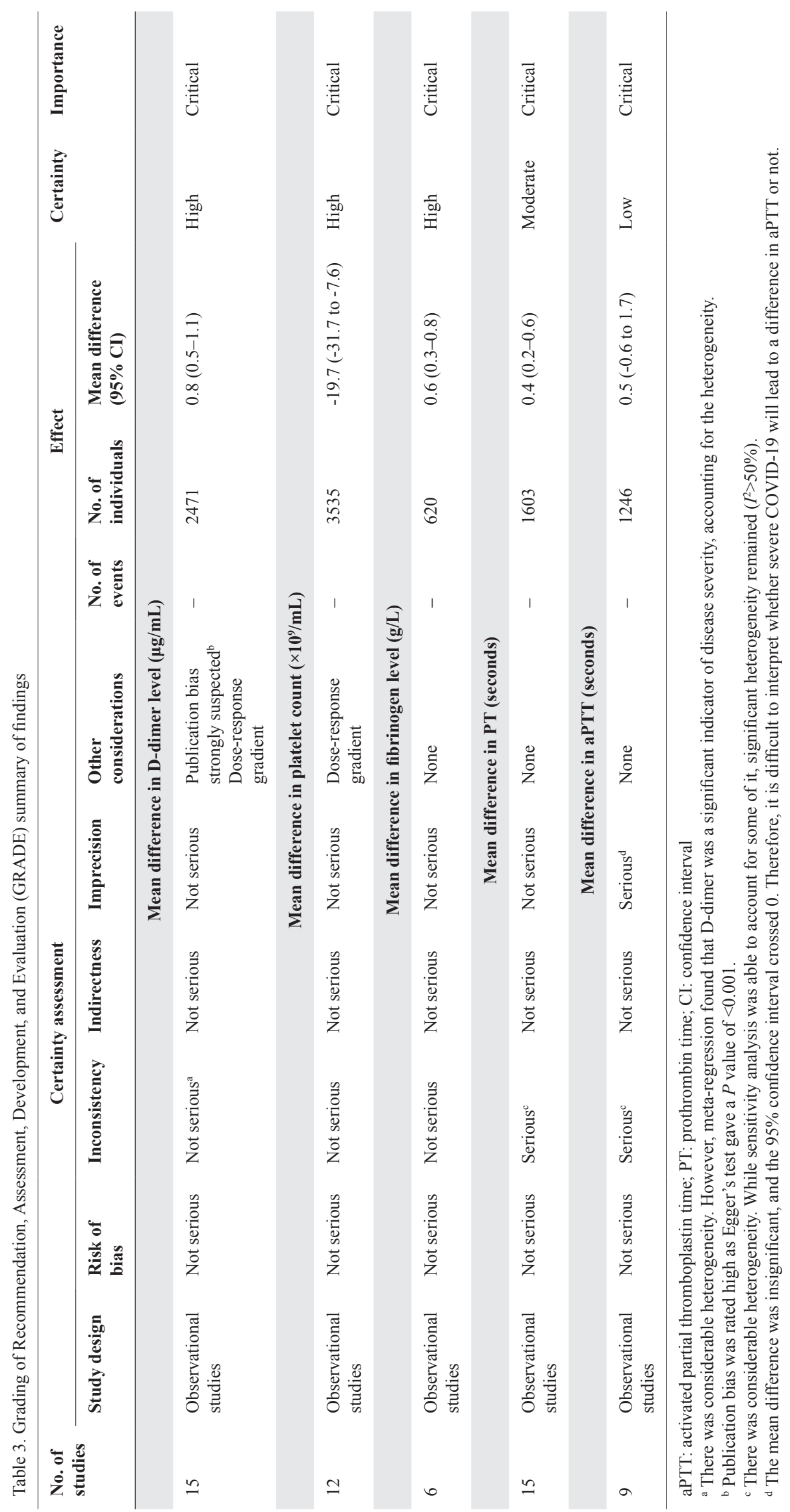


Table 4. Mechanisms of coagulopathy in COVID-19 patients with therapeutic options

\begin{tabular}{|c|c|c|c|}
\hline No. & Possible mechanisms of coagulopathy in COVID-19a & Target molecule(s) & Proposed therapeutic options \\
\hline 1 & $\begin{array}{l}\text { Virus-induced endothelial dysfunction resulting in } \\
\text { upregulation of von Willebrand factor, toll-like receptor } \\
\text { activation, and tissue factor pathway activation, leading } \\
\text { to formation of cross-linked fibrin clots. }{ }^{58}\end{array}$ & Coagulation factors & $\begin{array}{l}\text { Unfractionated heparin or } \\
\text { low-molecular-weight heparin } \\
\text { Mild disease: prophylactic dose } \\
\text { Severe disease: full dose }\end{array}$ \\
\hline 2 & $\begin{array}{l}\text { Increased plasminogen level in patients with comorbidities } \\
\text { is associated with increase ability of the virus to bind to } \\
\text { ACE2R. }{ }^{5} \text { This binding facilitates viral entry and } \\
\text { accentuates the endothelial injury. }\end{array}$ & $\begin{array}{l}\text { Plasminogen, S protein, } \\
\text { ACE2R }\end{array}$ & $\begin{array}{l}\text { a. Soluble ACE2 } \\
\text { b. Spike vaccine } \\
\text { c. ACE2R blockers } \\
\text { d. ACE inhibitors } \\
\text { e. Aprotinin } \\
\text { f. Heparin (binds to S protein) }\end{array}$ \\
\hline 3 & $\begin{array}{l}\text { Increased plasminogen level is also associated with } \\
\text { hyperfibrinolysis and D-dimer formation. } .^{5} \text { The process } \\
\text { propagates clot formation, entangles platelets and } \\
\text { contributes to consumptive coagulopathy. }\end{array}$ & Plasminogen & Aprotinin \\
\hline 4 & $\begin{array}{l}\text { Severe COVID-19 cases might have an increased } \\
\text { predilection for activation of both alternative and } \\
\text { lectin-based complement pathways. This activation } \\
\text { leads to endothelial dysfunction and prothrombotic states. }{ }^{59}\end{array}$ & Complement C5 & Eculizumab \\
\hline 5 & $\begin{array}{l}\text { Thrombin can initiate thrombosis and proinflammatory } \\
\text { responses by virtue of its procoagulant characteristic. }{ }^{60} \\
\text { May be responsible for localised thrombogenic } \\
\text { manifestations. }\end{array}$ & Thrombin & Recombinant antithrombin \\
\hline 6 & $\begin{array}{l}\text { Cytokine storm may itself potentiate endothelial injury } \\
\text { and activate coagulation cascade. }\end{array}$ & Interleukin 6 & $\begin{array}{l}\text { Tocilizumab, heparin, } \\
\text { Cytosorb, plasmapheresis. } \\
\text { (Heparin downregulates } \\
\text { interleukin-6 level. }{ }^{58} \text { ) }\end{array}$ \\
\hline 7 & $\begin{array}{l}\text { Antiphospholipid antibodies have been implicated in } \\
\text { thrombotic events. }{ }^{50}\end{array}$ & Lupus anticoagulants & $\begin{array}{l}\text { Heparin to be used judiciously with strict } \\
\text { monitoring of anti-factor Xa levels }\end{array}$ \\
\hline
\end{tabular}

ACE: angiotensin-converting enzyme; ACE2: angiotensin-converting enzyme 2; ACE2R: angiotensin-converting enzyme 2 receptor; COVID-19: coronavirus disease 2019

a Superscript numbers refer to reference numbers in REFERENCES

is required. Concordant with our findings, recent reviews on thrombo-inflammatory and haematological biomarkers have also concluded that patients with severe COVID-19 manifest hypercoagulable conditions (e.g. elevated D-dimer and fibrinogen levels) as well as a drop in platelet count. ${ }^{61,62}$

Our systematic review has certain limitations and results should hence be interpreted with caution. The analysis was based mainly on retrospective or cohort studies with significant heterogeneity during the early pandemic. Most of the initial studies on CIC were from China. The random-effects model was used when conducting this meta-analysis for the anticipated heterogeneity, in addition to using the GRADE approach to rate the certainty of evidence. We did additional subgroup analysis to account for heterogeneity. Furthermore, the meta-regression analyses were constrained by an inherent lack of power that increased the risk of type II errors. We included the studies of non-survivors in those with severe disease; however, it is possible that some patients with severe disease might have survived. Another potential limitation is the inability to accurately determine the timing at which coagulation results were being used in the publications for this review. Finally, Egger's test yielded nonsignificant results for most of our primary endpoints, except for D-dimer studies that had significant publication bias. Nonetheless, the Joanna Briggs Institute appraisal of the included studies suggested that they were of high quality, limiting the possibility of publication bias. The GRADE system showed low to high level of certainty for the results of the analysis.

\section{CONCLUSION}

This systematic review and meta-analysis demonstrated significant variability of the coagulation parameters in non-severe versus severe COVID-19. COVID-19 patients manifest a dynamic coagulation profile with the progression of disease severity. Both platelet count and D-dimer level significantly correlated with the severity 
of disease. CIC represents a spectrum of clinical manifestations ranging from prothrombotic stage to consumptive coagulopathy, depending on the disease severity. Diligent monitoring of routine coagulation parameters (platelet count, PT, D-dimer and fibrinogen) may be helpful to titrate the need for anticoagulation in COVID-19 patients. Further research should focus on the mechanisms of the derangement of coagulation in COVID-19. Understanding the mechanisms would then enable selection of the most appropriate diagnostic tests and scoring systems as well as help physicians choose optimal therapies for coagulopathy manifested during different stages of COVID-19.

\section{Acknowledgement}

We thank Ms Suei Nee Wong of the medical library of NUS Yong Loo Lin School of Medicine for her assistance with the formulation of the search strategy.

\section{REFERENCES}

1. Giannis D, Ziogas IA, Gianni P. Coagulation disorders in coronavirus infected patients: COVID-19, SARS-CoV-1, MERS-CoV and lessons from the past. J Clin Virol 2020;127:104362.

2. Sharma S, Keswani P, Bhargava A, et al. Overview of early cases of coronavirus disease 2019 (COVID-19) at a tertiary care centre in North India. Ann Acad Med Singap 2020;49:449-55.

3. Griffin DO, Jensen A, Khan M, et al. Pulmonary embolism and increased levels of d-dimer in patients with coronavirus disease. Emerg Infect Dis 2020;26:1941-3.

4. Mao L, Jin H, Wang M, et al. Neurologic manifestations of hospitalized patients with coronavirus disease 2019 in Wuhan, China. JAMA Neurol 2020;77:683-90.

5. Ji HL, Zhao R, Matalon S, et al. Elevated plasmin(ogen) as a common risk factor for COVID-19 susceptibility. Physiol Rev 2020;100:1065-75.

6. Henry BM, de Oliveira MHS, Benoit S, et al. Hematologic, biochemical and immune biomarker abnormalities associated with severe illness and mortality in coronavirus disease 2019 (COVID-19): A meta-analysis. Clin Chem Lab Med 2020; 58:1021-8.

7. Lippi G, Plebani M, Henry BM. Thrombocytopenia is associated with severe coronavirus disease 2019 (COVID-19) infections: A meta-analysis. Clin Chim Acta 2020;506:145-8.

8. World Health Organization. Clinical management of severe acute respiratory infection when novel coronavirus (nCoV) infection is suspected: Interim guidance, 25 January 2020. Available at: https://apps.who.int/iris/bitstream/handle/10665/330854/WHO-nCoVClinical-2020.2-eng.pdf? sequence $=1 \&$ isAllowed $=y$. Accessed on 1 October 2020.

9. National Health Commission. Diagnosis and treatment protocols for patients with novel coronavirus pneumonia (trial version 5, revised), 4 February 2020. Available at: http://www.kankyokansen. org/uploads/uploads/files/jsipc/protocol_V5.pdf. Accessed on 1 October 2020

10. National Health Commission \& National Administration of Traditional Chinese Medicine. Diagnosis and treatment protocol for novel coronavirus pneumonia (trial version 7). Chin Med J (Engl) 2020;133:1087-95.
11. DerSimonian R, Laird N. Meta-analysis in clinical trials. Control Clin Trials 1986;7:177-88

12. Wan X, Wang W, Liu J, et al. Estimating the sample mean and standard deviation from the sample size, median, range and/or interquartile range. BMC Med Res Methodol 2014;14:135.

13. Guyatt GH, Oxman AD, Vist GE, et al. GRADE: an emerging consensus on rating quality of evidence and strength of recommendations. BMJ 2008;336:924-6.

14. Guyatt GH, Oxman AD, Kunz R, et al. GRADE guidelines, 7. Rating the quality of evidence-Inconsistency. J Clin Epidemiol 2011; 64:1294-302.

15. Iorio A, Spencer FA, Falavigna M, et al. Use of GRADE for assessment of evidence about prognosis: Rating confidence in estimates of event rates in broad categories of patients. BMJ 2015;350:h870.

16. GRADEpro GDT. Available at: https://gradepro.org. Accessed on 10 October 2020

17. Guyatt G, Oxman AD, Akl EA, et al. GRADE guidelines: 1. Introduction - GRADE evidence profiles and summary of findings tables. J Clin Epidemiol 2011;64:383-94.

18. Santesso N, Carrasco-Labra A, Langendam M, et al. Improving GRADE evidence tables part 3: Detailed guidance for explanatory footnotes supports creating and understanding GRADE certainty in the evidence judgments. J Clin Epidemiol 2016;74:28-39.

19. Bonetti G, Manelli F, Patroni A, et al. Laboratory predictors of death from coronavirus disease 2019 (COVID-19) in the area of Valcamonica, Italy. Clin Chem Lab Med 2020;58:1100-5.

20. Cui S, Chen S, Li Xi, et al. Prevalence of venous thromboembolism in patients with severe novel coronavirus pneumonia. J Thromb Haemost 2020;18:1421-4.

21. Fogarty H, Townsend L, Cheallaigh CN, et al. COVID19 coagulopathy in Caucasian patients. Br J Haematol 2020;189:1044-9.

22. Fu J, Kong J, Wang W, et al. The clinical implication of dynamic neutrophil to lymphocyte ratio and D-dimer in COVID-19: A retrospective study in Suzhou China. Thromb Res 2020;192:3-8.

23. Gao Y, Li T, Han M, et al. Diagnostic utility of clinical laboratory data determinations for patients with the severe COVID-19. J Med Virol 2020;92:791-6.

24. Helms J, Tacquard C, Severac F, et al. High risk of thrombosis in patients with severe SARS-CoV-2 infection: A multicenter prospective cohort study. Intensive Care Med 2020;46:1089-98.

25. Klok FA, Kruip MJHA, van der Meer NJM, et al. Incidence of thrombotic complications in critically ill ICU patients with COVID-19. Thromb Res 2020;191:145-7.

26. Li J, Xu G, Yu H, et al. Clinical characteristics and outcomes of 74 patients with severe or critical COVID-19. Am J Med Sci 2020; 360:229-35.

27. Liu J, Li S, Liu J, et al. Longitudinal characteristics of lymphocyte responses and cytokine profiles in the peripheral blood of SARS-CoV-2 infected patients. EBioMedicine 2020;55:102763.

28. Liu Y, Liao W, Wan L, et al. Correlation between relative nasopharyngeal virus RNA load and lymphocyte count disease severity in patients with COVID-19. Viral Immunol 10 April 2020.

29. Lv Z, Cheng S, Le J, et al. Clinical characteristics and co-infections of 354 hospitalized patients with COVID-19 in Wuhan, China: A retrospective cohort study. Microbes Infect 2020;22:195-9.

30. Panigada M, Bottino N, Tagliabue $P$, et al. Hypercoagulability of COVID-19 patients in intensive care unit: A report of thromboelastography findings and other parameters of hemostasis. J Thromb Haemost 2020;18:1738-42. 
31. Qu R, Ling Y, Zhang YH, et al. Platelet-to-lymphocyte ratio is associated with prognosis in patients with coronavirus disease-19. J Med Virol 2020;92:1533-41.

32. Sun S, Cai X, Wang H, et al. Abnormalities of peripheral blood system in patients with COVID-19 in Wenzhou, China. Clin Chim Acta 2020;507:174-80.

33. Tabatabai A, Rabin J, Menaker J, et al. Factor VIII and functional protein $\mathrm{C}$ activity in critically ill patients with coronavirus disease 2019: A case series. A A Pract 2020;14:e01236.

34. Tang N, Bai H, Chen X, et al. Anticoagulant treatment is associated with decreased mortality in severe coronavirus disease 2019 patients with coagulopathy. J Thromb Haemost 2020;18:1094-9.

35. Wan S, Xiang Y, Fang W, et al. Clinical features and treatment of COVID-19 patients in northeast Chongqing. J Med Virol 2020; 92:797-806.

36. Wu C, Chen X, Cai Y, et al. Risk factors associated with acute respiratory distress syndrome and death in patients with coronavirus disease 2019 pneumonia in Wuhan, China. JAMA Intern Med 2020;180:934-43

37. Yang X, Yang Q, Wang Y, et al. Thrombocytopenia and its association with mortality in patients with COVID-19. J Thromb Haemost 2020;18:1469-72.

38. Yao Q, Wang $\mathrm{P}$, Wang $\mathrm{X}$, et al. A retrospective study of risk factors for severe acute respiratory syndrome coronavirus 2 infections in hospitalized adult patients. Pol Arch Intern Med 2020;130:390-9.

39. Zhang $\mathrm{G}, \mathrm{Hu} \mathrm{C}$, Luo $\mathrm{L}$, et al. Clinical features and short-term outcomes of 221 patients with COVID-19 in Wuhan, China. J Clin Virol 2020;127:104364.

40. Zhang G, Zhang J, Wang B, et al. Analysis of clinical characteristics and laboratory findings of 95 cases of 2019 novel coronavirus pneumonia in Wuhan, China: A retrospective analysis. Respir Res 2020;21:74.

41. Zhang JJ, Dong X, Cao YY, et al. Clinical characteristics of 140 patients infected with SARS-CoV-2 in Wuhan, China. Allergy 2020;75:1730-41.

42. Zhao X, Wang K, Zuo P, et al. Early decrease in blood platelet count is associated with poor prognosis in COVID-19 patientsIndications for predictive, preventive, and personalized medical approach. EPMA J 2020;11:1-7.

43. Zheng $\mathrm{Y}, \mathrm{Xu} \mathrm{H}$, Yang $\mathrm{M}$, et al. epidemiological characteristics and clinical features of 32 critical and 67 noncritical cases of COVID-19 in Chengdu. J Clin Virol 2020;127:104366.

44. Zou Y, Guo H, Zhang Y, et al. Analysis of coagulation parameters in patients with COVID-19 in Shanghai, China. Biosci Trends 2020; $14: 285-9$.

45. Ribes A, Vardon-Bounes F, Mémier V, et al. Thromboembolic events and COVID-19. Adv Biol Regul 2020;77:100735.
46. Huang C, Wang Y, Li X, et al. Clinical features of patients infected with 2019 novel coronavirus in Wuhan, China. Lancet 2020; 395:497-506.

47. Liu W, Tao ZW, Wang L, et al. Analysis of factors associated with disease outcomes in hospitalized patients with 2019 novel coronavirus disease. Chin Med J (Engl) 2020;133:1032-8.

48. Tang N, Li D, Wang X, et al. Abnormal coagulation parameters are associated with poor prognosis in patients with novel coronavirus pneumonia. J Thromb Haemost 2020;18:844-7.

49. Harzallah I, Debliquis A, Drénou B. Lupus anticoagulant is frequent in patients with COVID-19. J Thromb Haemost 2020;18:2064-5.

50. Zhang Y, Xiao M, Zhang S, et al. Coagulopathy and antiphospholipid antibodies in patients with COVID-19. N Engl J Med 2020;382:e38.

51. Connors JM, Levy JH. Thromboinflammation and the hypercoagulability of COVID-19. J Thromb Haemost 2020; 18:1559-61.

52. Marini JJ, Gattinoni L. Management of COVID-19 respiratory distress. JAMA 2020;323:2329-30.

53. Glowacka I, Bertram S, Herzog P, et al. Differential downregulation of ACE2 by the spike proteins of severe acute respiratory syndrome coronavirus and human coronavirus NL63. J Virol 2010;84:1198-205.

54. Esmon CT. The interactions between inflammation and coagulation. Br J Haematol 2005;131:417-30.

55. Camerer E, Kolstø AB, Prydz H. Cell biology of tissue factor, the principal initiator of blood coagulation. Thromb Res 1996; $81: 1-41$

56. Iba T, Levy JH. Sepsis-induced coagulopathy and disseminated intravascular coagulation. Anesthesiology 2020;132:1238-45.

57. Paranjpe I, Fuster V, Lala A, et al. Association of treatment dose anticoagulation with in-hospital survival among hospitalized patients with COVID-19. J Am Coll Cardiol 2020;76:122-4.

58. Atallah B, Mallah SI, AlMahmeed W. Anticoagulation in COVID-19. Eur Heart J Cardiovasc Pharmacother 2020;6:260-1.

59. Magro C, Mulvey JJ, Berlin D, et al. Complement associated microvascular injury and thrombosis in the pathogenesis of severe COVID-19 infection: A report of five cases. Transl Res 2020;220:1-13.

60. Kowalewski M, Fina D, Słomka A, et al. COVID-19 and ECMO: The interplay between coagulation and inflammation-A narrative review. Crit Care 2020;24:205.

61. Asghar M, Hussain N, Shoaib H, et al. Hematological characteristics of patients in coronavirus 19 infection: A systematic review and meta-analysis. J Community Hosp Intern Med Perspect 2020; 10:508-13.

62. Chaudhary R, Garg J, Houghton DE, et al. Thrombo-inflammatory biomarkers in COVID-19: Systematic review and meta-analysis of 17,052 patients. Mayo Clin Proc Innov Qual Outcomes 8 Feb 2021. 